\title{
artigo
}

\section{Vigilância em Saúde na pandemia de COVID-19 e os desafíos do SUS na atualidade}

\author{
Health Surveillance in the COVID-19 pandemic and the challenges of SUS in the current scenario \\ Vigilancia en Salud en la pandemia COVID-19 y los desafíos del SUS en la actualidad
}

\begin{abstract}
RESUMO
Objetivo: descrever e discutir sobre os desafios do SUS na atualidade e a atuação da Vigilância em Saúde no contexto da pandemia do novo coronavírus. Método: Ensaio teórico ancorado na literatura científica nacional e internacional, acrescida da análise crítica dos autores. Com base na construção teórica sobre o pensar reflexivo, foram abordados, a Vigilância em Saúde no contexto da epidemia do novo coronavírus; e os desafios do SUS na contemporaneidade. Resultados: 0 maior desafio na atualidade continua sendo político, e resistir aos ataques e riscos de desmantelamento do SUS pelas políticas de ajuste fiscal. Outros desafios incluem, os interesses econômicos e financeiros ligados às empresas de saúde; proposta político-ideológica da Cobertura Universal em Saúde; desfinanciamento; insuficiência da infraestrutura pública; reprodução do modelo médico hegemônico. Conclusão: Espera-se que a pandemia de COVID-19 desperte reflexões na população em relação à repolitização da sociedade em defesa do SUS enquanto Sistema Universal de Saúde.
\end{abstract}

DESCRITORES: COVID-19; SARS-CoV2; Sistema Único de Saúde; Epidemiologia; Saúde Pública; Vigilância em Saúde Pública;

\section{ABSTRACT}

Objective: to describe and discussing the challenges of SUS in the current scenario and also the role of Health Surveillance in the context of the new coronavirus pandemic. Method: Theoretical essay anchored in national and international scientific literature, plus authors' critical analysis. Based on the theoretical construction on reflective thinking, the following were discussed, the Health Surveillance in the context of the epidemic of the new coronavirus; and the challenges of SUS in contemporary times. Results: The biggest challenge nowadays remains political and resisting the attacks and risks of dismantling the SUS due to fiscal adjustment policies. Other challenges including, the economic and financial interests linked to healthcare companies; political proposal for Universal Health Coverage; divestment; insufficient public infrastructure; reproduction of the hegemonic medical model. Conclusion: It is expected that the COVID-19 pandemic will arouse reflections in the population regarding repoliticization of society in defense of SUS as a UHS.

DESCRIPTORS: COVID-19; SARS-CoV2; Unified Health System; Epidemiology; Public Health; Health Surveillance.

\section{RESUMEN}

Objetivo: describir y discutir los desafíos del SUS en el escenario actual y el rol de la Vigilancia en Salud en el contexto de la pandemia de coronavirus. Método: Ensayo teórico anclado en la literatura científica nacional e internacional, más análisis crítico de los autores. A partir de la construcción teórica sobre el pensamiento reflexivo, se discutió, la Vigilancia de la Salud en el contexto de la epidemia del COVID-19; y los desafíos del SUS en la actualidad. Resultados: El mayor desafío en la actualidad sigue siendo político y resistir los ataques y riesgos de desmantelar el SUS por las políticas de ajuste fiscal. Otros desafíos incluyen, los intereses económicos y financieros vinculados a las empresas de salud; propuesta política de Cobertura Universal de Salud; despojo; infraestructura pública insuficiente; reproducción del modelo médico hegemónico. Conclusión: Se espera que la pandemia COVID-19 suscite reflexiones en la población sobre la repolitización de la sociedad en defensa del SUS como Sistema de Salud Universal.

DESCRIPTORES: COVID-19; SARS-CoV2; Sistema Único de Salud; Epidemiología; Salud Pública; Vigilancia de la Salud.

RECEBIDO EM: 19/01/2021 APROVADO EM: 03/02/2021

\section{Luís Carlos Lopes-Júnior}

Enfermeiro. Especialista em Gestão em Saúde e Especialista em Onoclogia. Doutor em Ciências pela Universidade de São Paulo (USP). Professor Adjunto do Departamento de Enfermagem, Centro de Ciências da Saúde, Universidade Federal do Espírito Santo - UFES. Área: Saúde Coletiva. Vitória, Espírito Santo, Brasil.

ORCID: 0000-0002-2424-6510 


\section{Ariane Araújo Lacerda}

Acadêmica de Enfermagem e Obstetrícia na Universidade Federal do Espírito Santo - UFES, Vitória, Espírito Santo, Brasil. ORCID: 0000-0003-3468-7034

\section{Flávia Fonseca Venâncio}

Acadêmica de Enfermagem e Obstetrícia na Universidade Federal do Espírito Santo - UFES, Vitória, Espírito Santo, Brasil. ORCID: 0000-0001-8309-4353

\section{Leticia Peisino Buleriano}

Acadêmica de Enfermagem e Obstetrícia na Universidade Federal do Espírito Santo - UFES, Vitória, Espírito Santo, Brasil. ORCID: 0000-0003-4231-5417

\section{Letícia Baltar Sobreira}

Acadêmica de Enfermagem e Obstetrícia na Universidade Federal do Espírito Santo - UFES, Vitória, Espírito Santo, Brasil. ORCID: 0000-0002-3206-3554

\section{INTRODUÇÃO}

$\mathbf{R}$ esultante de um conjunto de esforços em defesa da democracia provenientes do movimento de Reforma Sanitária Brasileira, em 1988 foi promulgada a Constituição Federal Cidadã que trazia as bases para o maior sistema de saúde público do mundo - o Sistema Único de Saúde (SUS) -, que deve ser compreendido não como política de governo ou de partido, mas sim, como uma política de Estado capaz de garantir o direito e o acesso universal à saúde ${ }^{(1,2,3)}$. Esse marco constitucional-legal favoreceu a luta política em torno da construção do SUS, regulamentado por meio das Leis Orgânicas de Saúde 8.080/1990 e 8.142/1990, e teve efeito protetor no sistema mesmo em períodos em que a agenda neoliberal ganhou força, como na década de $1990^{(4)}$.

A análise da situação atual do SUS é uma tarefa que demanda, em primeiro lugar o reconhecimento da complexidade do processo político, do desenvolvimento organizacional e da reorientação dos processos de trabalho nos vários níveis de gestão do sistema. Existe uma tendência à diversificação das estratégias utilizadas pelos dirigentes do sistema em cada conjuntura e esfera de governo, em um processo contínuo de ajuste das propostas às possibilidades de ação e dos constrangimentos decorrentes da permanente negociação com os diversos atores políticos envolvidos, seja os que atuam internamente ao sistema (gestores, profissionais/trabalhadores de saúde), seja os que pressionam o sistema de fora, buscando que as decisões adotadas atendam a seus interesses, demandas e necessidades $^{(2,3,5-9)}$. Com isso, o SUS se apresenta em uma arena permanente de conflitos, negociações, pactos, com os quais se tenta, na maioria das vezes, administrar crises e introduzir reformas em aspectos parciais de sua estrutura organizacional e político gerencial ${ }^{(1,8)}$.

Em número especial do periódico The Lancet dedicado ao SUS pesquisadores reafirmaram que o principal problema do SUS é político ${ }^{(8)}$. Daí decorrem vários outros problemas, com destaque para o subfinanciamento, na medida em que se mantém um reduzido gasto público em função da prioridade dada às políticas de ajuste fiscal e de crescimento econômico e competitividade. Em última instância há que se considerar que o SUS demanda sobretudo, uma busca por sustentabilidade tanto institucional quanto política ${ }^{(8,9)}$. Estudo recente publicado avaliando os 30 anos da existência do SUS, os autores destacaram as contribuições significativas no acesso aos serviços de saúde, o que resultou em reduções das desigualdades dos indicadores de saúde e em melhoria da equidade ${ }^{(2)}$.

Todavia, atualmente, mesmo depois de 32 anos da sua criação, ainda que reconhecidos seus avanços, Paim (2019)(1) ressalta os principais obstáculos e ameaças ao SUS, quais sejam: a) limitadas bases sociais e políticas; $b$ ) interesses econômicos e financeiros ligados às empresas de saúde; c) proposta político-ideológica da Cobertura Universal em Saúde; d) desfinanciamento; e) insuficiência da infraestrutura pública; e) reprodução do modelo médico hegemônico ${ }^{(10)}$.

Apesar da existência de tantos desafios, o SUS se mostra eficiente. Um ponto a destacar é a ação da Vigilância em Saúde que foi essencial na detecção dos casos de microcefalia e na associação causal com a síndrome congênita do Zika Vírus, bem como, atualmente, frente à pandemia do novo coronavírus, atuando na identificação, controle, manejo adequado e prevenção dos casos de Covid-19. É através dela que o SUS foi organizado para receber os casos identificados, buscando garantir a integralidade e longitudinalidade do cuidado assim como os fluxos e contrafluxos entre os serviços na Rede de Atenção à Saúde (RAS) $)^{(11)}$.

A Secretaria de Vigilância em Saúde (SVS), instituída no ano de 2003 pelo Ministério da Saúde (MS), nasce como um modelo para atuar como suporte político-institucional para o processo de descentralização e de reorganização dos serviços e das práticas de saúde, trabalhando em conjunto com a Agência Nacional de Vigilância Sanitária (Anvisa) na formulação das políticas de vigilância em saúde e políticas sanitárias ${ }^{(12)}$. Em 2006, no Brasil, o Pacto pela Saúde estabeleceu as Diretrizes Nacionais da Vigilância em Saúde e propôs como seus componentes as ações de vigilância, promoção, prevenção e controle de doenças e agravos à saúde. Ainda, o 
conceito engloba a vigilância e o controle das doenças transmissíveis; a vigilância das doenças e agravos não transmissíveis; a vigilância da situação de saúde, vigilância ambiental em saúde, vigilância da saúde do trabalhador e a vigilância sanitária ${ }^{(13)}$. Posteriormente, a Portaria No 1.378 de 9 de julho de 2013, é promulgada a fim de regulamentar as responsabilidades e define as diretrizes para execução e financiamento das ações de Vigilância em Saúde pela União, Estados, Distrito Federal e Municípios, relativos ao Sistema Nacional de Vigilância em Saúde e Sistema Nacional de Vigilância Sanitária ${ }^{(14)}$.

Nos últimos anos, um importante avanço na área de vigilância foi a publicação da Política Nacional de Vigilância em Saúde - aprovada por meio da Resolução do Conselho Nacional de Saúde no 588 no ano de 2018 - que a estabelece como política pública de Estado essencial ao SUS, de responsabilidade exclusiva do poder público e possui como finalidade definir os princípios, as diretrizes e as estratégias a serem observados pelas três esferas de gestão do SUS. A Resolução define a Vigilância em Saúde como um processo contínuo e amplo de coleta, análise e compartilhamento de dados relacionados à saúde, objetivando a implementação de medidas de saúde pública para proteção e promoção da saúde e prevenção de agravos e doenças ${ }^{(15)}$. Nesse contexto, questionou-se "Como tem sido a atuação da Vigilância em Saúde no enfrentamento a pandemia da COVID-19 e quais os desafios do SUS na contemporaneidade? Para tanto, objetivou-se neste ensaio teórico, descrever e discutir sobre os desafios do SUS na atualidade e a atuação da Vigilância em Saúde no contexto da pandemia do novo coronavírus.

\section{MÉTODO}

Trata-se de um ensaio teórico, cuja fundamentação baseia-se na formulação discursiva acerca da temática, sustentado na literatura científica nacional e internacional e análise crítica dos autores. Para tanto, realizou-se um levantamento da literatura científica $^{(16)}$ contemporânea que foi posteriormente, submetidos ao Método de Leitura Científica: visão sincrética do texto; visão analítica; visão sintética ou leitura interpretativa ${ }^{(17)}$. Com base na construção teórica sobre o pensar reflexivo, ${ }^{(18)}$ foram abordados: I) a Vigilância em Saúde no contexto da epidemia do novo coronavírus no Brasil; e II) Desafios do SUS na contemporaneidade.

\section{RESULTADOS E DISCUSSÃO}

Vigilância em Saúde no contexto da epidemia do novo coronavírus no Brasil

No ano de 2020, frente à pandemia de COVID-19 inúmeras reflexões foram levantadas sobre os sistemas de saúde e sistemas de vigilância ao redor do globo ${ }^{(19-21)}$. A Organização Mundial da Saúde (OMS), em 30 de janeiro de 2020, declarou a COVID-19 como uma Emergência de Saúde Pública de Importância Internacional, e, em 11 de março de 2020, como uma pandemia $^{(22)}$. No Brasil, medidas foram adotadas antes do primeiro caso ser confirmado. Em 22 de janeiro, iniciaram-se as ações do Centro de Operações de Emergência do Ministério da Saúde (MS), coordenado pela SVS/MS. Nesse primeiro momento, as ações buscaram promover a informação e comunicação para a população, além de capacitação para os profissionais de saúde e a expansão da cobertura do SUS, especialmente na atenção terciária, com o aumento de leitos de unidades de terapia intensiva, respiradores, equipamentos de proteção individual ${ }^{(23)}$. Em 20 de março de 2020, o MS declarou a transmissão comunitária da COVID-19 e, assim, iniciou a adoção de medidas para a mitigação de sua transmissão, como isolamento e distanciamento social ${ }^{(24)}$.

Ressalta-se que o sistema de vigilância do Brasil apresentou uma resposta rápida: no dia 26 de fevereiro de 2020 foi confirmado o primeiro caso da COVID-19 no país, no dia 03 de março já havia dois casos confirmados e 488 casos suspeitos notificados. Em janeiro, antes mesmo do primeiro caso confirmado no país, a SVS/MS acionou os Pontos Focais
Nacionais do Regulamento Sanitário Internacional da OMS (PFN-RSI/OMS), emitiu boletins epidemiológicos, disponibilizou números diários sobre os casos (suspeitos, confirmados e descartados), bem como frequentes entrevistas e comunicados à imprensa e a população sobre a pandemia ${ }^{(25)}$. Dados mais recentes mostram 8.488.099 casos confirmados apenas no Brasil e 209.847 mortes por Covid-19 em 18 de janeiro de $2021^{(26)}$.

Vale ressaltar que a comunicação e disseminação das informações epidemiológicas, pelos boletins epidemiológicos e orientações das possíveis formas de prevenção sobre a doença através das mídias sociais é essencial para a conscientização da população e para que essa seja participante ativa e corresponsável do seu cuidado ${ }^{(27)}$.

Sendo assim, é por meio da Vigilância em Saúde que ocorre a busca ativa de casos, a testagem oportuna e a captação de contatos que garantem a identificação precoce da doença e a possível quebra na cadeia de transmissão. Além disso, $80 \%$ dos casos leves de Covid-19 e grande parcela dos casos moderados procuram a Atenção Primária à Saúde (APS) como o primeiro acesso para o cuidado, reafirmando o papel desse nível de atenção como protagonista do cuidado, cumprindo seu papel de ordenadora da rede e coordenadora do cuidado somando-se a atenção secundária e terciária cumprindo seus papéis determinados na RAS, para atendimento e manejo dos casos garantindo condiçôes para um cuidado eficaz e de qualidade ${ }^{(28)}$.

Outro aspecto que deve ser destacado é que dentre as recomendações das agências sanitárias regulatórias nacionais e internacionais foram propostas a aceleração no desenvolvimento de vacinas, medidas terapêuticas e diagnósticos ${ }^{(29)}$. No que concerne a corrida pelo desenvolvimento de uma vacina, cerca de 200 projetos de desenvolvimento estão registrados na OMS, dos quais 13 estão ou concluíram a fase 3 para a avaliação de eficácia, a última etapa antes da aprovação pelas agências reguladoras e posterior imunização da população ${ }^{(30)}$.

$\mathrm{Na}$ busca por garantir mais doses para a 
população brasileira, três acordos de transferência de tecnologia foram assinados no país: um do Instituto de Tecnologia em Imunobiológicos da Fundação Oswaldo Cruz (Bio-Manguinhos/Fiocruz)/Ministério da Saúde com o laboratório AstraZeneca ${ }^{(31)}$ que está trabalhando em parceria com a Universidade Oxford (Reino Unido), que estabeleceu o fornecimento inicial de 100 milhões de doses; outro do Instituto Butantan do Estado de São Paulo com a empresa Sinovac, China (Coronavac) ${ }^{(32)}$, garantindo o fornecimento de 46 milhões de doses; e o último do Instituto de Tecnologia do Paraná (TECPAR) do Estado do Paraná com o Instituto Gamaleya, Rússia $(\text { Sputinik V) })^{(33)}$.

As empresas Pfizer e Moderna e o Instituto Gamaleya divulgaram preliminarmente os resultados dos estudos da fase 3 , na qual a eficácia das suas vacinas apresentou resultados acima de $90 \%$, sem efeitos adversos graves, demonstrando também a segurança destes imunobiológicos. Os resultados da análise de eficácia primária provisória da AstraZeneca apontam para uma eficácia de $62,1 \%$ para os participantes que receberam duas doses padrão e de $90 \%$ para aqueles que receberam primeiro meia dose e após um mês uma dose completa. A vacina do Instituto Butantan do Estado de São Paulo contra a Covid-19 obteve $50,38 \%$ de eficácia global no estudo desenvolvido no Brasil ${ }^{(32)}$. Esses resultados são promissores uma vez que neste momento de urgência a OMS definiu que uma vacina com proteção acima de 50\% será aceitável ${ }^{(34)}$.

A Diretoria Colegiada da Agância Nacional de Vigilância Sanitária (Anvisa) aprovou no dia 17 de janeiro de 2021, por unanimidade, a autorização temporária de uso emergencial da vacina CoronaVac, desenvolvida pela farmacêutica Sinovac em parceria com o Instituto Butantan, e da vacina Covishield, produzida pela farmacêutica Serum Institute of India, em parceria com a AstraZeneca/Universidade de Oxford/Fiocruz ${ }^{(35)}$.

Tanto a Fiocruz quanto o Instituto Butantan devem dar continuidade aos estudos e à geração de dados para per- mitir o registro sanitário na Anvisa. Da mesma forma, ambos precisam manter o monitoramento da segurança das vacinas, fundamental para garantir que os benefícios continuem a superar os riscos para as pessoas que recebem vacinas contra Covid-19. A Anvisa, como órgão regulador federal, pode revisar e ajustar as condições para o uso emergencial frente a resultados que sejam considerados relevantes, inclusive dados e informações provenientes de autoridades reguladoras internacionais ${ }^{(35)}$. No dia seguinte, 18/1/2021, o país já começou a receber as primeiras doses para serem utilizadas no território nacional e se prepara para o início da Campanha de Vacinação contra o novo coronavírus com esses dois imuniobiológicos.

Esse será outro grande desafio para a Vigilância em Saúde, uma vez que, apesar de toda a aceleração do desenvolvimento vista até o momento, ainda existem muitas lacunas no conhecimento, impondo algumas dificuldades para se organizar o plano de vacinação. A maioria das vacinas que chegou à fase 3 terá um esquema vacinal com duas doses, devendo ser aplicadas entre 14 a 29 dias após a aplicação da primeira dose $\mathrm{e}^{(29)}$, o que exigirá um enorme esforço e organização dos serviços de saúde para garantir a adesão do elevado contingente populacional a ser vacinado em um curto prazo, paras as duas doses.

Também exigirá a identificação da pessoa vacinada nos postos de vacinação, havendo a necessidade da criação de um sistema nominal que seja simplificado e que consiga inserir os dados de forma oportuna, para acompanhar a evolução da vacinação e que seja integrado ao Sistema de Informações do Programa Nacional de Imunizações (SIPNI) do MS. Ao mesmo tempo, será necessária a implementação da vigilância de eventos adversos pós-vacinação ativa e de forma oportuna, visando a garantir a segurança da vacinação durante todo o processo. Outro importante monitoramento que deverá ser realizado, após o início da vacinação, será o das gestantes que forem vacinadas inadvertidamente, ou seja, no momento da vacinação não sabiam que já estavam grávidas, portanto, deverão ser acompanhadas para avaliar a segurança da vacinação no período gestacional ${ }^{(36)}$.

Ainda, será necessária a definição e priorização dos grupos a serem vacinados, elencados com base no risco de adoecer, ter complicações e óbito: portadores de doenças crônicas, diabetes, doenças cardiovasculares, câncer, doença renal, doença respiratória, enfermidades hematológicas, obesidade e pessoas idosas (acima de 60 anos). Os profissionais de saúde, por estarem na linha de frente do cuidado dos pacientes com COVID-19, devem ser os primeiros a ser vacinados. Outros grupos deverão ser incluídos na estratégia de vacinação na medida em que houver disponibilidade de vacinas como os indígenas, quilombolas, população ribeirinha e privada de liberdade, professores, entre outros trabalhadores considerados como essenciais $^{(36)}$.

Vale ressaltar que o SUS possui o Programa Nacional de Imunizações (PNI), coordenado pelo Ministério da Saúde, de forma compartilhada com as Secretarias Estaduais e Municipais de Saúde, o qual vem se consolidando como uma das mais relevantes intervenções em saúde públi$\mathrm{Ca}^{(37)}$. Criado em 1973, em sua trajetória de 46 anos, o PNI tem uma história de conquistas e desafios a ser contada. Caracteriza-se como uma política pública eficiente, impactando cada vez mais no perfil de morbimortalidade da população brasileira, adequando-se às mudanças ocorridas nos campos político, epidemiológico e social ${ }^{(37)}$. De fato, o PNI tem larga experiência em organizar campanhas de vacinação em massa, atingindo elevadas coberturas vacinais e o seu objetivo que é proteger a saúde da população definida nessas estratégias. Ao se atingir elevadas coberturas vacinais, além de reduzir casos de doença na população-alvo estabelecida para a vacinação, contribui para a diminuição da circulação de agentes infecciosos nas comunidades, impactando positivamente na saúde daqueles que não serão vacinados, uma vez que passam a estar protegidos indiretamente (imunidade coletiva ou de rebanho) ${ }^{(38-39)}$. 


\section{Desafios do SUS na contempora- neidade}

Apesar de descrito na Constituição de 1988, nenhum governo assumiu o SUS como prioridade política a ser consolidado como um sistema de saúde universal ${ }^{(4)}$, fato reiterado pela publicação do Decreto $\mathrm{n}^{\circ} 7.508$ de 2011, que regulamentou a Lei 8.080/90 apenas após 21 anos da sua promulgação ${ }^{(40)}$.

O SUS sofreu sérios obstáculos no seu desenvolvimento histórico diante do subfinanciamento crônico de anos, e ainda enfrenta, na contemporaneidade, ameaças à sua consolidação e o risco de desmonte ante as políticas econômicas ultraliberais no plano interno e, internacionalmente, diante da proposta de Cobertura Universal em Saúde (do inglês, Universal Health Coverage-UHC) e da ação política dos que defendem sistemas de saúde orientados para o mercado ${ }^{(1)}$.

Nas últimas décadas, acirrou-se o debate internacional sobre diferentes concepções de universalidade em saúde, polarizado nas propostas de Sistema Universal de Saúde (Universal Health System-UHS) versus Cobertura Universal em Saúde (UHC). Sabe-se que para países em desenvolvimento, o termo UHC é empregado em referência à cobertura por serviços básicos, ou por cobertura de seguros de saúde, públicos ou privados, indicando ênfase no subsídio à demanda, em detrimento da construção de sistemas públicos universais $^{(41-43)}$.

UHC é um termo ambíguo que tem levado a diferentes interpretações e abordagens por parte das autoridades sanitárias nacionais, organizações governamentais e não governamentais, especialmente nos países em desenvolvimento. A proposta de UHC é ancorada em três pilares: i) foco no financiamento por combinação de fundos (pooling); ii) afiliação por modalidade de asseguramento; iii) definição de cesta limitada de serviços, com vistas à redução do papel do Estado, restringindo-o à regulação do sistema de saúde ${ }^{(42)}$. Já o UHS é financiado por fundos públicos a partir da receita de impostos gerais e contribuições sociais, o que proporciona maior solidariedade, redistribuição e equidade. $\mathrm{O}$ grau de redistribuição depende da progressividade da carga tributária, isto é, maior proporção de tributação sobre rendas e propriedade do que sobre consumo ${ }^{(42)}$.

Embora a APS é preconizada como estratégia para a cobertura universal, todavia, pode ter significados muito distintos. Na agenda UHC, APS refere-se a um pacote básico de serviços e medicamentos essenciais definidos em cada país, correspondendo a uma abordagem seletiva para alcance de um universalismo básico em países em desenvolvimento. Distingue-se da abordagem integral dos sistemas públicos universais onde corresponde à base do sistema e deve ordenar a rede assistencial ${ }^{(42-43)}$.

Nesse sentido a proposta de UHC atenderia às demandas de seguradoras brasileiras e, possivelmente, do capital financeiro e seguradoras internacionais por ampliação de participação no 'mercado de saúde ${ }^{\mathrm{s}(42)}$. O fortalecimento adicional do setor privado representa a maior ameaça aos sistemas de saúde universais como no caso do SUS, e ao direito universal à saúde. A proposta de UHC é pouco clara quanto a seus pressupostos e estratégias. A utilização de conceitos e termos semelhantes aos previstos para os sistemas universais dificulta o entendimento das mudanças arquitetadas em curso.

Claramente, o Brasil ilustra de modo contundente as tensões na construção e na defesa de um sistema de saúde universal, sobretudo, considerando as suas desigualdades sociais e de saúde, a situação de país capitalista periférico, a crise econômica e política, as fragilidades institucionais, o crescimento de ideologias conservadoras e neoliberais, bem como as ameaças à democracia. Portanto, unidade, agilidade e efetividade continuam fundamentais para a militância em defesa do SUS, da democracia e do projeto civilizatório da $\mathrm{RSB}^{(1)}$.

Apesar das conquistas significativas, os problemas, obstáculos e desafios enfrentados pelo SUS nas três últimas décadas adquirem ainda maior relevância diante das crises econômica e política desde 2014, especialmente decorrente das consequências do golpe parlamentar-midiático de 2016 e dos resultados das eleições presidenciais de 2018. Assim, o SUS não foi consolidado como um sistema de saúde universal, tal como proposto pela RSB e assegurado pela Constituição ${ }^{(1)}$.

Salienta-se ainda, a agenda neoliberal que se instalou no país nos últimos anos, principalmente, com as medidas de austeridade fiscais implementadas em 2016 (Emenda Constitucional 95 - um novo regime fiscal com um limite para os gastos do governo federal, que vigorará pelos próximos 20 anos $^{(4)}$ ou seja, até 2036, sendo o teto fixado para 2020 correspondente ao orçamento disponível para os gastos de 2019, acrescido da inflação daquele ano principalmente para os gastos com saúde, educação e políticas sociais. Isso associado às novas políticas ambientais, educacionais e de saúde do atual governo brasileiro, tem ameaçado a sustentabilidade e capacidade do sistema de ofertar acesso universal para todos ${ }^{(2)}$. Em meio ao cenário emergencial pelo avanço da infecção pelo novo coronavírus no país, essas medidas de austeridades fiscais são postas em xeque ao evidenciar uma falta de estrutura e de capacidade do sistema de saúde de oferecer respostas sociais às necessidades de saúde da população.

Conforme estudo da Comissão de Orçamento e Financiamento (Cofin) do Conselho Nacional de Saúde (CNS), no fim de 2019 o prejuízo ao SUS já era de $\mathrm{R} \$ 20$ bilhões com a EC, antes da pandemia de COVID-19. Ao longo de duas décadas, os danos são estimados em $\mathrm{R} \$ 400$ bilhões - montante a menos para custear o SUS ${ }^{(45)}$, o que tem causado um sucateamento paulatino do SUS em vários pontos da Rede de Atenção à Saúde.

O subfinanciamento crônico do SUS é um desafio constante do sistema, e que durante todos esses anos ainda observamos: insuficiência de recursos, iniquidade nas condições de financiamento, gastos em saúde em nível subnacional e baixa participação dos investimentos no setor de saúde pública. Em contraparti- 
da, ocorre a persistência de altos gastos privados subsidiados pelo Estado, além da baixa prioridade econômica e fiscal do financiamento federal em saúde ${ }^{(4)}$. Atualmente, a participação pública na gestão de saúde no Brasil é pequena, equivalente a $41 \%$, quando comparada a de outros países, inclusive os EUA, que não possui um sistema universal de saúde e destina $45,5 \%$ do seu PIB à saúde; podemos citar também países da Europa, como a Itália e Reino Unido, que destinam $77,2 \%$ e $82 \%$ do seu PIB à saúde, respectivamente ${ }^{(8,40)}$.

Quanto à infraestrutura, o SUS sofre com a falta de recursos materiais e humanos e com a insuficiência de estabelecimentos e serviços. A APS - porta de entrada da RAS, vem sofrendo grandes ataques, como: o Previne Brasil - novo modelo de financiamento da APS com base em captação ponderada, a tentativa de desconstrução da Estratégia de Saúde da Família (ESF), com redução dos Agentes Comunitários de Saúde (ACS), extinção dos Núcleo Ampliado de Saúde da Família e Atenção Básica (NASF-AB) e perda de profissionais com o encerramento do Programa Mais Médicos, que dificultam o acesso da população ao sistema de saúde $e^{(1,8,40,46)}$.

\section{CONCLUSÃO}

O Brasil não tem sido um bom exemplo no combate ao novo coronavírus, mesmo com a grandiosidade e importância do SUS e as ações de Vigilância em Saúde para controle da pandemia. Isso porque, a falta de um alinhamento, coesão e coordenação entre governadores de estados, presidente da república e a falta de um Ministro da
Saúde, somado ao negacionismo à ciência e desrespeito do presidente da república às orientações baseadas em evidências, de agências sanitárias reguladoras nacionais e internacionais, o que tem atrapalhado sobremaneira a controle da epidemia no nosso país.

O maior desafio do SUS na contemporaneidade continua sendo político, isto é, atravessar a tormenta, resistindo aos ataques e riscos de desmantelamento do SUS pelas políticas de ajuste fiscal. Ainda que o cenário político atual aposte em investimentos no setor privado em detrimento ao fortalecimento de políticas públicas, espera-se que a pandemia de COVID-19 desperte reflexões na população em relação à politização da sociedade em defesa do SUS enquanto Sistema Universal de Saúde e não como Cobertura Universal em Saúde.

\section{REFERÊNCIAS}

1. Paim JS. Os sistemas universais de saúde e o futuro do Sistema Único de Saúde (SUS). Saúde Debate. 2019;43(spe5):15-28.

2. Castro MC, Massuda A, Almeida G, Menezes-Filho NA, Andrade MV, de Souza Noronha KVM, et al. Brazil's unified health system: the first 30 years and prospects for the future. Lancet. 2019;394(10195):345-356.

3. Lopes-Júnior LC. Policies, epidemiology, and praxis in Brazil's Unified National Health System. Cad Saude Publica.2020;36(11):e00295120.

4. Machado CV, Lima LD, Baptista TWF. Health policies in Brazil in times of contradiction: paths and pitfalls in the construction of a universal system. Cad Saude Publica. 2017;33Suppl 2(Suppl 2):e00129616.

5. Massuda A, Hone T, Leles FAG, de Castro MC, Atun R. The Brazilian health system at crossroads: progress, crisis and resilience. BMJ Glob Health. 2018;3(4):e000829.

6. Barreto ML, Rasella D, Machado DB, Aquino R, Lima D, Garcia $\mathrm{LP}$, et al. Monitoring and evaluating progress towards Universal Health Coverage in Brazil. PLoS Med. 2014;11(9):e1001692.

7. Watts J. Brazil's health system woes worsen in economic crisis. Lancet. 2016;387(10028):1603-4.

8. Paim J, Travassos C, Almeida C, Bahia L, Macinko J. The Brazilian health system: history, advances, and challenges. Lancet. 2011;377(9779):1778-97.

9. Bolina AF, Bomfim E, Lopes-Júnior LC. Frontline Nursing care: the COVID-19 pandemic and the Brazilian Health System. SAGE Open Nurs. 2020;6:2377960820963771.
10. Paim JS. Sistema Único de Saúde (SUS) aos 30 anos. Ciênc. Saúde Colet. 2018; 23(6)1723-1728.

11. Brasil, Ministério da Saúde. Coronavírus COVID-19: guia de vigilância epidemiológica emergência de saúde pública de importância nacional pela doença pelo coronavírus 2019. 1 ed. Brasília/DF: Ministério da Saúde; 2020.

12. Oliveira CM, Cruz MM. Sistema de Vigilância em Saúde no Brasil: avanços e desafios. Saúde Debate. 2015;39(104):255267.

13. Brasil, Ministério da Saúde. Diretrizes Nacionais da Vigilância em Saúde. Ed. 1. Brasília: Ministério da Saúde; 2010.

14. Brasil, Ministério da Saúde. Portaria N 1.378 de 9 de julho de 2013. Brasília; 2013.

15. Brasil, Ministério da Saúde. Resolução Nº 588, de 12 de juIho de 2018. Institui a Política Nacional de Vigilância em Saúde. Brasília; 2018.

16. Vosgerau DSAR, Romanowski JP. Estudos de revisão: implicações conceituais e metodológicas. Rev Diálogo Educ. 2014;14(41):165-189.

17. Cervo A, Bervian PA. Metodologia científica. 5ed. São Paulo: Prentice Hall, 2002.

18. Souza RA. The foundations of pedagogy by John Dewey:a reflection on pragmatist epistemology. Rev Contraponto Eletrônica. 2012;12(2):227-233.

19. Silva Junior FJGD, Sales JCES, Monteiro CFS, Monteiro TAS, Lima RAG, Lopes-Junior LC. Impact of COVID-19 pandemic on mental health of young people and adults: a sys- 


\section{REFERÊNCIAS}

tematic review protocol of observational studies. BMJ Open. 2020;10(7):e039426.

20. Lopes-Júnior LC, Bomfim E, Silveira DSC, Pessanha RM, Schuab SIPC, Lima RAG. Effectiveness of mass testing for control of COVID-19: a systematic review protocol. BMJ Open. 2020;10(8):e040413.

21. Lopes-Júnior LC. A Saúde Coletiva no epicentro da pandemia de COVID-19 no Sistema Único de Saúde. Saúde Coletiva. 2020;10(56):3080-3084.

22. Mahase E. Covid-19: WHO declares pandemic because of "alarming levels" of spread, severity, and inaction. BMJ 2020;368:m1036.

23. Oliveira WK, Duarte E, França GVA, Garcia LP. Como - Brasil pode deter a COVID-19. Epidemiol Serv Saúde. 2020;29(2):e2020044.

24. Sales CMM, Silva AL, Maciel ELN. Vigilância em saúde da COVID-19 no Brasil: investigação de contatos pela atenção primária em saúde como estratégia de proteção comunitária. Epidemiol Serv Saúde. 2020;29(4):2020373.

25. Croda JHR, Garcia LP. Immediate Health Surveillance Response to COVID-19 Epidemic. Epidemiol Serv Saude. 2020;29(1):e2020002.

26. Coronavirus Resource Center. Johns Hopkins University; [citado 18 de janeiro de 2021]. https://coronavirus.jhu.edu/ (acessado em 18/Jan/2021).

27. Santos JG dos, Mota FPB. A transparência governamental em tempos de Covid-19: reflexões do quadro brasileiro. Gestão Sociedade .;14(39):3716-24. Gestão E Sociedade, 14(39), 37163724.

28. Medina MG, Giovanella L, Bousquat A, Mendonça MHM, Aquino R; Comitê Gestor da Rede de Pesquisa em Atenção Primária à Saúde da Abrasco. Primary healthcare in times of COVID-19: what to do? Cad Saude Publica. 2020;36(8):e00149720.

29. World Health Organization. Timeline: WHO response COVID-19. https://www.who.int/emergencies/diseases/novel-coronavirus-2019/interactive-timeline (acessado em 30/ Dez/2020).

30. World Health Organization. Draft landscape of COVID-19 candidate vaccines - 2 December 2020. https://www.who.int/ publications/m/item/draft-landscape-of-covid-19-candidatevaccines (acessado em 30/Dez/2020).

31. Portal Fiocruz. Covid-19: Fiocruz firmará acordo para produzir vacina da Universidade de Oxford. https://portal.fiocruz.br/ noticia/covid-19-fiocruz-firmara-acordo-para-produzir-vacina-da-universidade-de-oxford(acessado em 30/Dez/2020).

32. Instituto Butantan. Vacina contra a COVID-19. https://vacinacovid.butantan.gov.br/vacinas (acessado em 30/Dez/2020).

33. Tecpar: Instituto de Tecnologia do Paraná. Vacina russa será submetida à Anvisa até o fim de setembro. http:// www.tecpar.br/Noticia/Vacina-russa-sera-submetida-Anvisa-ate-o-fim-de-setembro (acessado em 30/Dez/2020).
34. Ansede M, Galocha A, Oliveira R. As diferenças abismais entre as vacinas de Oxford, Pfizer e Moderna, a Coronavac e a Sputnik V. El País 2020; 24 nov.

35. Anvisa. Anvisa aprova por unanimidade uso emergencial das vacinas. https://www.gov.br/anvisa/pt-br/assuntos/noticias-anvisa/2021/anvisa-aprova-por-unanimidade-uso-emergencial-das-vacinas (acessado em 18/Jan/2021).

36. Domingues CMAS. Challenges for implementation of the COVID-19 vaccination campaign in Brazil. Cad Saude Publica. 2021;37(1):e00344620.

37. Domingues CMAS, Teixeira AMS. Coberturas vacinais e doenças imunopreveníveis no Brasil no período 1982-2012: avanços e desafios do Programa Nacional de Imunizações. Epidemiol Serv Saúde 2013;22(1):9-27.

38. Rochel de Camargo Jr Kenneth. Here we go again: the reemergence of anti-vaccine activism on the Internet. Cad. Saúde Pública. 2020;36( Suppl 2):e00037620.

39. Domingues CMAS, Maranhão AGK, Teixeira AM, Fantinato FFS, Domingues RAS. The Brazilian National Immunization Program: 46 years of achievements and challenges. Cad Saude Publica. 2020;36Suppl 2(Suppl 2):e00222919.

40. Lopes-Júnior LC. Desafios do SUS na contemporaneidade. In. Silva Júnior FJG, Sales JCS, Galiza FT, Monteiro CFS, organizadores. Políticas, epidemiologia e experiências no Sistema Único de Saúde (SUS): possibilidades e desafios do cenário brasileiro. Curitiba: Editora CRV; 2020.

41. Noronha JC. Universal health coverage: how to mix concepts, confuse objectives, and abandon principles. Cad Saude Publica. 2013;29(5):847-9.

42. Giovanella L, Mendoza-Ruiz A, Pilar ACA, Rosa MCD, Martins GB, Santos IS, et al. Universal health system and universal health coverage: assumptions and strategies. Cien Saude Colet. 2018;23(6):1763-1776.

43. Lima, LD. Crisis y neoliberalismo: desafíos y alternativas políticas para la construcción de sistemas universales de salud en América Latina. Cad. Saúde Pública. 2017;33/ Suppl 2):e00047517.

44. Brasil. Governo do Brasil. Emenda Constitucional n. 95. Altera o Ato das Disposições Constitucionais Transitórias, para instituir o Novo Regime Fiscal. 2016. http://www.planalto.gov.br/ccivil_03/ constituicao/emendas/emc/emc95.htm (acessado em 23/Dez/2020).

45. Conselho Nacional de Saúde. Ministério da Saúde. Mais SUS menos Coronavirus: CNS intensifica campanha pela revogação da EC 95/2016. 2020. March 14. http://conselho.saude.gov.br/ ultimas-noticias-cns/1068-maissusmenoscoronavirus-cns-intensifica-campanha-pela-revogacao-da-ec-95-2016 (acessado em 23/Dez/2020).

46. Silva WMF, Ruiz JLS. A centralidade do SUS na pandemia do coronavírus e as disputas com o projeto neoliberal. Physis. 2020;30(3):e300302. 